\section{¿ABAJO EL SECRETO, VIVA LA PRIVACIDAD! DILEMAS MEDIÁTICOS EN EL ASUNTO SNOWDEN}

\author{
José María Muñoz Terrón \\ Universidad de Almería \\ ORCID iD: https://orcid.org/0000-0002-0584-1840 \\ jmterron@ual.es
}

\section{DOWN WITH SECRECY! LONG LIVE PRIVACY! MEDIA DILEMMAS IN THE SNOWDEN AFFAIR}

Cómo citar este artículo/Citation: Muñoz Terrón, J. M. (2020). ¡Abajo el secreto, viva la privacidad! Dilemas mediáticos en el asunto Snowden. Arbor, 196 (797): a573. https://doi. org/10.3989/arbor.2020.797n3010
Copyright: (C) 2020 CSIC. Este es un artículo de acceso abierto distribuido bajo los términos de la licencia de uso y distribución Creative Commons Reconocimiento 4.0 Internacional (CC BY 4.0).
RESUMEN: Este artículo es un análisis de tres relatos periodísticos de la filtración de documentos de la Agencia Nacional de Seguridad (NSA) de Estados Unidos, llevada a cabo por Edward Snowden: un libro, una película y una entrevista. El objetivo es sacar a la luz algunos dilemas éticos y políticos que subyacen al caso. Primero, una tensión paradójica entre la exigencia de transparencia frente al secreto estatal y la necesidad del secreto personal para el derecho a la privacidad, que refleja en nuestros tiempos la dialéctica publicidad / secreto en la modernidad temprana. Segundo, la ética hack-activista enfrenta los conflictos entre anonimato y visibilidad. Tercero, los periodistas en que Snowden confía afrontan el dilema entre los deberes de la información y la protección de la confidencialidad de las fuentes. El asunto Snowden es, pues, una oportunidad para extraer algunas lecciones sobre la ética de la comunicación en la era digital.

PALABRAS CLAVE: Ética; política; esfera pública; periodismo; transparencia; secreto; privacidad; vigilancia; filtración.
ABSTRACT: This article is an analysis of three journalistic accounts of the leaking of documents from the United States National Security Agency, done by Edward Snowden: a book, a movie, and an interview. The goal is to bring to light some ethical and political dilemmas underlying the case. First, a paradoxical tension between the demand for transparency versus the state's secrecy and the need of personal secrecy for the right to privacy, which nowadays reflects the publicity versus secrecy dialectic in early modernity. Second, the hack-activist ethic facing the conflicts between anonymity and visibility. Third, the journalists trusted by Snowden confront the dilemma between the obligations of information and the protection of source confidentiality. The Snowden affair is thus an opportunity to learn some lessons about the ethics of communication in the digital age.

KEYWORDS: Ethics; politics; public sphere; journalism; transparency; secrecy; privacy; surveillance; leaks. 


\section{INTRODUCCIÓN: ¿POR QUÉ ME IMPORTA A MÍ EL ASUNTO SNOWDEN?}

«I used to work for the government. Now I work for the public»

@Snowden

«Solía trabajar para el gobierno. Ahora trabajo para el público». Este lacónico tuit de Edward Snowden, publicado en diciembre de 2014 (https://twitter.com/ snowden?lang=de), condensa toda una trayectoria, que replicaría, con todas sus paradojas, en los actuales tiempos de los medios digitales, el giro que se produjo en la conciencia política de un típico ciudadano burgués del dieciocho, de mentalidad ilustrada y vocación cosmopolita. Si entendemos que con gobierno se refiere metonímicamente al Estado, y con público a la sociedad civil o ciudadanía, el declarado cambio de lealtades de Snowden contiene implícita una destacable crítica política. Un "gobierno»-aunque sea democrático y goce de la presunción de legitimidad del Estado de Derecho- no siempre trabaja para «el público» al que se debe. La denuncia de traición por parte de su gobierno, de la que es objeto el revelador de información clasificada, pasaría a recaer así directamente sobre el acusador, el Estado, al que justamente se le reprocha ahora actuar, con determinadas medidas y procedimientos, en contra de la sociedad.

En este artículo se analiza la privacidad como un tópico fundamental en torno al que se construye, por parte de los medios, los profesionales de la comunicación social y el propio Edward Snowden, el asunto de las filtraciones de documentos y de prácticas secretas de vigilancia masiva de las agencias de inteligencia y los gobiernos de Estados Unidos, presentado como una noticia que conectaría con los intereses de cualquier individuo del mundo. La presuposición es que prácticamente toda persona se sentirá afectada si le hablan de que alguien puede interferir en su esfera privada, cualquiera se sentirá molesta o irritada si detecta que es espiada, vigilada, controlada en su vida particular. La privacidad es el punto crucial con el que los medios y el propio Edward Snowden presentan su caso como tema de interés y relevancia pública a escala mundial. También desde las ciencias sociales, la ética y la política se apunta igualmente que lo que está en juego en las filtraciones del, ahora famoso, exempleado de la Agencia Nacional de Seguridad (en adelante, NSA, por las siglas en inglés) de Estados Unidos, en esta ascendente sociedad de la vigilancia generalizada (Bauman y Lyon, 2013), son las libertades civiles, los derechos humanos, la privacidad (Lyon, 2015, p. VIIII, pp. 91-114 y pp. 158-163). Como ha sido estudiado en relación con otros hechos contemporáneos, hay un importante papel de los medios en la construcción de los así denominados asuntos públicos como problemas, así como en su conversión en eventos mediáticos (Arquembourg, 1998; Arquembourg; 2009). La función de mediación o intermediación de los media, como cauces o vehículos de las revelaciones, fue clave desde el principio en el proceder de Snowden, de forma muy contrastada con el papel secundario y subordinado que la profesión periodística desempeñara en las filtraciones de Wikileaks, aunque en ambos casos los medios ejercieran de narradores de las historias que dan sentido a los documentos desvelados (Landert y Miscione, 2017).

Las líneas de demarcación entre lo secreto, que es desacreditado, y lo privado, que es exaltado, son sin embargo más difusas de lo que sugieren los planteamientos de Snowden y los periodistas que elaboran el relato de sus revelaciones: esto es, relaciones de oposición al secreto (gubernamental), como sinónimo de control, vigilancia, y en favor de la privacidad personal, como sinónimo de libertad. Muy al contrario, como el título de este artículo -y el propio planteamiento del asunto Snowden en los medios- pretende reflejar, hay latente todo un complejo juego de contraposiciones múltiples entre estos cuatro conceptos: 'secreto', 'transparencia', 'privacidad', 'publicidad'. En primer lugar, 'transparencia' se opone a 'secreto', si se piensa en términos de activismo social frente a la opacidad de los Estados; 'privacidad' y 'publicidad' también se contraponen, pero como ámbitos que se necesitan uno a otro (Arendt, 1958/1998; Benhabib, 1996, pp. 211-215). En segundo lugar, 'transparencia' también puede ser opuesto a 'privacidad', cuando son los poderes políticos, gubernamentales, tecnológicos o mediáticos los que fuerzan a las personas a ser transparentes. La invasión de la esfera personal, íntima, familiar, privada, o la demanda de que todo sea visible para todos, pueden hacerse en nombre de los superiores intereses del pueblo, de la nación o del Estado; pero también desde las múltiples instancias de vigilancia internalizadas en la nueva era digital (Han, 2014, pp. 99-103; Whitaker, 1999, pp. 218-251). Así mismo, los medios, en su afán informativo, rozan en ocasiones los límites del respeto a las vidas privadas. La libertad de información, el imperativo de la publicidad, interpretados en clave de transparencia incondicional pueden ser vividos por las personas como una amenaza, también en sociedades con libertades de expresión, información e investigación.

Tres documentos periodísticos se emplean en esta investigación para analizar las tensiones entre 'secreto', 
'transparencia', 'privacidad' y 'publicidad' en los discursos de justificación de las filtraciones de documentos de la NSA llevadas a cabo por Snowden. De ellos, dos son audiovisuales: uno cinematográfico, la película CitizenFour, producida por D. Wilutzky y dirigida por Laura Poitras, que testimonia en formato de reportaje documental cómo se desarrolla el proceso de contacto y las conversaciones entre el filtrador y los periodistas que publican las primeras revelaciones; el otro, televisivo y digital, la entrevista concedida por Snowden, refugiado en ese momento en Rusia, al programa El Objetivo, del canal privado español La Sexta, no en la versión abreviada y montada para su emisión (el 13 de marzo de 2016) por la cadena, sino el archivo con la conversación íntegra de la periodista Ana Pastor con Snowden, puesto a disposición general del público en Internet. El tercer documento analizado es el libro No Place to Hide del periodista Glenn Greenwald (2014a), un extenso relato en primera persona de cómo vivió este informador, en tanto profesional de los medios preocupado por los derechos humanos, su relación con Edward Snowden, siendo uno de los periodistas escogidos por él para hacer de intermediario de las filtraciones.

Las conclusiones del análisis de estos materiales se exponen a continuación, siguiendo estas cuatro líneas de reflexión: 1.a) el asunto Snowden refleja en nuestra era digital la tensión dialéctica en la que se gestaba en los inicios de la Modernidad el principio ilustrado de la publicidad contra el secretismo estatal; de ahí que 2.a) el actual activismo en defensa de la privacidad frente a las injerencias de la vigilancia estatal asuma una ambivalente posición frente al secreto y sus técnicas, que se rechazan cuando lo ejercen los Estados, al tiempo que se lo busca cuando la ciudadanía comprometida desea mantener cierta reserva sobre acciones; por su parte, 3. ${ }^{a}$ ) también la profesión periodística, a la que Snowden refrenda con su confianza, en su hoy cuestionado papel de intermediadora de la información, afronta sus propios dilemas entre la obligación de proteger la confidencialidad de las fuentes y el mandato de la información. Finalmente 4. a) se apunta a la necesidad de extraer algunas lecciones de este asunto para la ética de la comunicación en la era digital.

\section{DILEMAS DE LA ESFERA PÚBLICA: ENTRE EL SECRETO Y LA TRANSPARENCIA}

\subsection{Frente a secreto (estatal), privacidad (personal) y transparencia (pública)}

El objetivo inicial de Snowden, según sus propias palabras, era «provocar un debate mundial sobre la privacidad, la libertad en Internet y los peligros de la vigilancia estatal». Y entre sus primeros propósitos para cuando se desvelase su identidad estaba difundir «un manifiesto a favor de la privacidad y en contra de la vigilancia para que lo firmase gente de todo el mundo y, de este modo, se hiciera patente la existencia de un movimiento global de apoyo a la protección de la privacidad». Para lo cual se muestra desde el principio dispuesto a asumir los riesgos y los costes personales precisos. Tan solo le preocupa, dice, «hacer todo esto por nada» (Greenwald, 2014b, p. 32). La exclusiva apelación a la defensa de la privacidad individual no habría bastado, sin embargo, al parecer, para atraer la atención general del público. Lo que convierte al tema Snowden en la gran historia periodística que se debe contar al mundo entero sería más bien el nexo entre la movilización del impulso de cualquier individuo para defender su privacidad y el interés morboso en el escándalo que suscita siempre la revelación de determinadas conductas que las gentes más poderosas quieren mantener en secreto. Desde su experiencia de escribir durante años en la prensa sobre la cuestión, sin demasiado eco, Greenwald observa que «la invasión de la privacidad y abuso de poder suelen verse como una abstracción, algo difícil de tomar de forma visceral. Es más, el tema de la vigilancia es siempre complejo, por lo que cuesta más implicar a la gente de forma generalizada». Pero... "Cuando se filtran documentos secretos, los medios prestan atención. Y el hecho de que el aviso lo diera alguien de dentro del aparato de seguridad nacional -y no un abogado de la American Civil Liberties Union o un defensor de las libertades civiles- seguramente suponía un refuerzo añadido». (Greenwald, 2014b, p. 33).

Esto nos lleva al centro mismo de uno de esos dilemas latentes en el asunto Snowden, la ambivalencia inherente a la tensión entre secreto y publicidad tal como se genera a partir de la moderna Ilustración (Hölscher, 1979; Koselleck, 1959). Para defender políticamente el derecho a la privacidad en la vida personal y las relaciones sociales, la actual ciudadanía cosmopolita digital necesita, como el emergente ciudadano burgués del XVII y el XVIII, cuestionar e incluso arrebatar a los Estados el "derecho al secreto". Las políticas de secretismo quedaron entonces identificadas con el absolutismo, que rechazaba el imperativo ilustrado de publicidad, lo mismo que el siglo XX ha dejado asociada la vigilancia secreta con los regímenes dictatoriales o totalitarios, aunque actualmente se practique en mayor o menor medida por toda clase de gobiernos, entidades y corporaciones. Junto a la justificación por la seguridad y la lucha antiterrorista, hay otros intereses menos declarables en la vigilancia 
y la monitorización de la población. Los documentos filtrados por Snowden llevan a Greenwald a afirmar que solo una parte de la vigilancia estaría dirigida a sospechosos de terrorismo. Tan importante o más era el espionaje económico y diplomático y una supervisión indiscriminada de la vida de poblaciones enteras. En suma, «el gobierno de EE.UU. había creado un sistema cuya finalidad era la completa eliminación de la privacidad electrónica en todo el mundo» (Greenwald, 2014b, p. 118). Por el contrario, la privacidad es un derecho cuya protección deben garantizar precisamente los Estados (Declaración Universal de los Derechos Humanos, artículo 12). De ahí lo políticamente intolerable de todo este perverso proceder estatal respecto del secreto y la privacidad: «El secretismo crea un espejo unidireccional: el gobierno de EE.UU. ve lo que hace todo el mundo incluida su propia población y nadie ve las acciones del gobierno. Es el desequilibrio supremo, el que permite la más peligrosa de las circunstancias humanas: el ejercicio de un poder ilimitado sin transparencia ni rendición de cuentas» (Greenwald, 2014b, p. 212). Hoy, gracias a las tecnologías electrónicas y digitales, la posibilidad de que el gobierno sepa cada vez más sobre lo que hace la ciudadanía, y esta sepa cada vez menos de lo que sus gobernantes hacen, presuntamente en su nombre en el mejor de los supuestos, es algo muy real. Por eso, se plantea hoy-como antes hizo la llustración-que justo lo contrario debería ser el caso: «La transparencia es para quienes tienen obligaciones públicas y ejercen el poder público. La privacidad para todos los demás.» (Greenwald, 2014b, p. 258)

Para conectar con el más amplio espectro de intereses del público, Snowden y los periodistas que le dan voz subrayan que las prácticas secretas de la NSA y del gobierno de Estados Unidos afectan a las vidas de cualesquiera personas del mundo, en sus derechos individuales, en particular el de protección de la privacidad. Aunque esta sea invocada en la polémica como principio constitucional propio de Estados Unidos más que como derecho humano universal, Snowden adquiere contornos de nuevo ciudadano digital cosmopolita modélico, en tanto es presentado como auténtico paladín de la defensa de la privacidad, convertida en sinónimo de libertad, frente al secreto o el arcano estatal, que de otra parte siempre ha estado en tensión con la libertad de información (Wegener, 2006). La causa de Snowden se inscribiría así en una secular "dialéctica", que desempeñó un papel crucial en la construcción de las esferas públicas de la Modernidad temprana en Europa (Hölscher, 1979), y en la que se contraponen, de una parte, el rechazo del secreto en política y la exigencia de publicidad desde las organizaciones de la sociedad civil, y de otra, la necesidad de una cierta discreción, reserva o secreto por parte de esas mismas asociaciones frente al Estado. En los documentos periodísticos analizados, tanto Snowden, como Greenwald o Poitras presentan la revelación de los documentos secretos de la NSA como un asunto de la máxima trascendencia para el público mundial, dado que en él estarían en juego a la vez los designios de la más alta política con la experiencia, que se presume elemental, del deseo de preservación de la intimidad de la vida personal. Frente al posible encogimiento de hombros de mucha gente que se preguntaría: '¿Por qué tendría yo que preocuparme, si no tengo nada que ocultar?', argumenta Snowden, que esto sería una comprensión errónea de la naturaleza del problema, la privacidad no tiene que ver con tener algo -o nada- que esconder:

«Historically [...] privacy is the foundation of all the rights [...] privacy is the fountainhead from of which all the rights are to right. It is the basis of freedom of speech because you haven't the right to say anything until you have the room intellectually to develop before [...] Similarly, freedom of religion wouldn't have meaning if you couldn't have space for yourself. To decide. What is your faith. What you believe in» (La Sexta, 2016, 13 de marzo, minuto 14).

Se trata, desde luego, de las libertades intelectuales de pensamiento y de expresión, pero más aún se trata de que la privacidad, como derecho que los Estados deben proteger, está implicada en gran cantidad de actos cotidianos, incluidos los relacionados con la adquisición de bienes en propiedad, una de las libertades más ensalzadas en la cultura política estadounidense. Snowden aguza la privacidad en su alegato, hasta convertirla en sinónimo de la libertad: "Privacy is really the word for liberty. It is the opportunity to have meaning for choices, to decide what you want to do, what you wonna say, what you want to share" (La Sexta, 2016, 13 de marzo, minuto 15; énfasis de E. Snowden) No se trata, pues, de algo de lo que quepa desentenderse sin poner en riesgo las posibilidades de cada persona de llegar a ser alguien y fiel a sí misma: "when you think [...] you don't care about privacy because you haven't anything to hide. It's not different to say I don't care about freedom of speech, because I have nothing to say. You don't care about freedom of press because you're not a journalist". En suma, concluye Snowden, existen derechos que te proporcionan valores importantes aunque tú no los estés desempeñando en este momento (La Sexta, 2016, 13 de marzo, minutos 15-16). 


\subsection{Dialéctica entre transparencia y secreto, publicidad y privacidad}

En términos políticos, el secreto ya fue cuestionado por la crítica ilustrada del absolutismo y de sus arcana imperii, aunque los finos análisis de R. Koselleck (1959) también pusieron de relieve la paradójica utilización del secreto por las sociedades masónicas, en la forma de la discreción o el silencio de los iniciados, justo como una estrategia frente a la falta de libertades de opinión y de expresión en los regímenes absolutos. En la actual controversia sobre las filtraciones de Snowden o de Wikileaks, el secreto de los procedimientos de vigilancia y control-con los que a menudo se viola precisamente el derecho a la privacidad- continúa apareciendo ante todo como un instrumento político de control por parte de los Estados y de los gobiernos, más usado cuanto más proclives son estos al autoritarismo, y menos a la defensa de la ciudadanía. Pues la reivindicación ilustrada es la publicidad y no una transparencia radical, indiscriminada, la opción del secreto para la gente de a pie no es una incoherencia hipócrita, como pretende denunciar Koselleck. En forma de discreción, de silencio o de reserva, el secreto (masónico) representaría para la ciudadanía ilustrada una paradójica exigencia frente a las restricciones de la libertad, frente al control y la vigilancia despóticos. En estos tiempos de ruido digital y mediático, la discreción y el silencio -el celo por el secreto- de los individuos pueden tener también un sentido ético-moral, ya sea desde un punto de vista psicosocial, como reivindica Georg Simmel, o contracultural, como apuntan David Le Breton o Paul Virilio (Callejo, 2003); e incluso un sentido cívicopolítico, pues para quienes se organizan frente a un poder tiránico o absoluto el secreto no es el de los gobernantes, el del "político" omnisciente platónico, el de la soberanía, sea monárquica o popular, que se coloca por encima de las leyes y los derechos, ni el arcano imperialista del gobernante absoluto o paternalista. Los secretos del poder despiertan -con razón- la desconfianza y el recelo de una población habituada a sospechar cuando el dominio se extralimita. Al mismo tiempo, sin embargo, cuando el principio normativo de la publicidad (existencia obligada de una esfera pública de comunicación libre) se excede hacia una exigencia incondicionada e incondicional de "transparencia" por la transparencia misma, en todos los niveles y aspectos de la vida social y política, también se debe hacer notar que la ejecución y la realizabilidad de las acciones políticas necesita una cierta discreción o secreto, sobre todo si aquellas conciernen a la seguridad de la ciudadanía (Hölscher, 1979). La historia del propio concepto de la esfera pública como ámbito efectivo y como norma política así lo muestra: a veces se necesita también de los consejos secretos y de los secretos de Estado (Wegener, 2006).

\section{DILEMAS DEL (HACK)ACTIVISMO POR LA PRIVACIDAD: ENTRE EL ANONIMATO Y LA VISIBILIDAD}

\subsection{Avatares del filtrador de secretos estatales y de- fensor de la privacidad individual}

El ciudadano Snowden experimenta en sus propias carnes una vertiginosa y paradójica transformación que va desde su pretendido papel inicial como filtrador anónimo, la clásica fuente confidencial periodística que permanece a salvo en su propia privacidad o secreto, hasta convertirse -en parte por la reacción del gobierno de Estados Unidos frente a las revelaciones, en parte por su propia voluntad, ya anticipada cuando decide entregar información a los medios- en un personaje público, de notoriedad mundial. Snowden deviene simultáneamente una de las caras más difundidas del momento en todos los medios y, a la par, en cuanto perseguido como traidor por el gobierno de su propio país, en un proscrito, compelido a permanecer constantemente oculto, a partir de aquella impersonal habitación de hotel en Hong Kong, donde da comienzo a sus filtraciones. Desposeído, pues, desde que inicia su aventura de revelador de secretos, de la posibilidad de tener un hogar, convertido en clandestino, y literalmente "privado" del derecho a un lugar propio, solo precariamente recuperado, gracias a ser acogido como refugiado en Moscú. De manera irónica Snowden viene a encarnar los múltiples significados implícitos en el título de la obra de Greenwald, sin un lugar donde esconderse. En la persona de Edward Joseph Snowden se cumple, de manera ejemplarizante quizá para el gobierno de Estados Unidos, la advertencia admonitoria contenida en las palabras del senador Frank Church con las que se abre el libro, que fueron pronunciadas, ien 1975 !, en su calidad de presidente del Comité del Senado para el estudio de las operaciones gubernamentales respecto de actividades de inteligencia. El enorme incremento de la capacidad tecnológica del gobierno permite controlar hasta tal punto los mensajes que circulan por el aire, que esto se podría volver contra el propio pueblo estadounidense, de modo que al final no habría privacidad para nadie en Estados Unidos ("no American would have any privacy left") y como consecuencia de este poder para controlar (to monitor) todas las comunicaciones, se llegaría al extremo de que "[T]here would be no place to hide", esto es, literalmente no habría (ningún) 
lugar donde esconderse, o también, no habría lugar donde esconder nada. Esta es la situación, cargada de paradójico simbolismo, en la que se coloca a sí mismo Snowden, con su defensa pública de la privacidad (individual), contra el secreto (estatal). Con un solo gesto se convierte en la perfecta encarnación de un ser típicamente mediático de pies a cabeza, y a la vez, por su entrada en la luz pública, en alguien despojado por completo de ese derecho a la privacidad por la que él ha emprendido su lucha; paralelamente constreñido en su derecho a (no) mostrarse e intervenir en público, hacer declaraciones, dar entrevistas en vivo (in flesh), al tiempo que su vida familiar e íntima es escrutada e invadida al máximo por el mismo aparato de vigilancia que él ha denunciado.

Desde este marco se entiende bien que Snowden se haya convertido en una suerte de héroe de la cultura más alternativa de los nuevos medios. Así es, por ejemplo, en un país como la actual Alemania que cuenta con un significativo movimiento de defensa de la libertad en Internet (el denominado Netzbewegung) y en el que la privacidad es un valor muy sensible, hasta el punto de que las vacilaciones a la hora de mantener su vigencia en el mundo digital actual, desde su defensa a ultranza hasta el coqueteo con la post-privacy, son en gran medida la clave de las fases que se pueden diferenciar en la evolución de este movimiento social por la privacidad en Internet, según muestra el análisis de los cambios en su discurso político (Ganz, 2015). Más allá de una cierta pose mesiánica del personaje, sobre la que se ha ironizado (Borradori, 2016), la experiencia del Ciudadano Snowden en el proceso que va desde su inicial papel de denunciante anónimo a su conversión en figura pública mundial "sin lugar donde esconderse", elevaría a la enésima potencia la tensión vivida por muchos activistas de la era digital. Estos se debaten a menudo en el dilema entre una visibilidad necesariamente ambivalente (deseada, por un lado, para mostrar públicamente la implicación personal en las protestas, pero que, al mismo tiempo, trae consigo una inevitable vulnerabilidad a la vigilancia estatal) y una anonimidad, también ambigua, necesaria para preservar la privacidad y el secreto personal de las comunicaciones y ejercer libremente su activismo, lejos de los controles de vigilancia estatales (Ballenthien et al., 2015; Freudenschuß, 2015). La existencia de un movimiento que se hace llamar Anonymous es suficientemente elocuente a este respecto. Este dilema parece bien gestionado en gran medida por el propio Snowden para sí mismo. En los documentos analizados, no resulta en cambio tan patente cómo lo asumirían, en su parte de responsabilidad, los profesionales de los medios, justo quizá porque la finalidad de su trabajo es precisamente la publicidad.

\subsection{Luchar contra el secreto con sus propios medios: ¿dilema o quimera?}

Entonces ¿la conjunción copulativa entre las dos consignas que dan título a este trabajo (¡Abajo el secreto! y iviva la privacidad!) es inevitable? Al contrario. Porque no es todo secreto el que se quiere eliminar en defensa de la privacidad. En el asunto Snowden y su tratamiento mediático, la reivindicación de la privacidad es inseparable del secreto, al menos en dos sentidos. Por una parte, el secreto es denigrable cuando se trata del secreto político de las prácticas de vigilancia masiva o de monitorización o control, llevadas a cabo desde las agencias estatales, que estarían obligadas a someterse al escrutinio público ciudadano, incluso a la transparencia; por otra parte, hablamos de secreto en un sentido distinto, cuando se trata, no de los Estados, sino de un derecho al secreto individual para la vida en sociedad y las relaciones interpersonales, cuyas funciones sociales fueron certeramente analizadas por Georg Simmel (1986, pp. 357-424). La sociología del secreto esbozada por Simmel (1993, pp. 317-323 y pp. 394-397), en sus textos sobre las comunicaciones privadas y la correspondencia epistolar fechados nada menos que en 1908, necesitaría ser repensada por completo en estos tiempos de intercambio postal casi exclusivo a través de mensajería electrónica, tan fácilmente interceptable y controlable por los sistemas de vigilancia, y tan fácilmente compartible a través de los medios digitales. En este sentido, el filtrador, como hackactivista, y los informadores que trabajan con él en la divulgación de los documentos secretos, necesitan ellos mismos del secreto (privado, particular) en las comunicaciones, tener claves seguras, dispositivos no conectados a Internet, cifrado o encriptación de las comunicaciones, intentar esquivar de todos los modos posibles la vigilancia a la que se saben sometidos, más incluso que cualesquiera otros ciudadanos. Resulta muy significativo que esta codificación se identifique a veces directamente con la misma noción de privacidad. Por ejemplo, le da nombre a uno de esos programas para las comunicaciones electrónicas seguras, PGP (pretty good privacy), del que el futuro filtrador de secretos de la NSA empieza a hablarle a Greenwald (2014b, p. 19) en el primer mensaje encriptado y anónimo que le dirige. En esto también Snowden responde a un cierto perfil, pues la búsqueda de las más variadas y efectivas prácticas de autoprotección de datos frente a la vigilancia generalizada se ha convertido para mu- 
chos activistas de los movimientos por los derechos en Internet, más que en un reto a sus habilidades tecnológicas, en una auténtica actividad política (Ochs, 2015), en la que cada cual ha de ser responsable para sí mismo y sus contactos, con la colaboración ocasional de personas expertas. Cabe plantearse, sin embargo, si tiene sentido que toda la ciudadanía de a pie quiera jugarse sus derechos en este juego del ratón y el gato, de espías y contraespías, en el propio terreno de las enormes capacidades tecnológicas que pueden desarrollar los Estados y las corporaciones. Snowden muestra una confianza tan fuerte en las técnicas de cifrado o encriptación para defender la privacidad de los individuos que parece olvidar la importancia básica de la más elemental y primaria confianza entre las personas, que desde luego él mismo está ejercitando con los profesionales de los medios que él ha escogido para publicar sus revelaciones. Esta, más que posible, deformación o vicio profesional de un ex trabajador de la NSA muestra su lado más exaltado, y quizá ingenuo, cuando en una carta a los periodistas glosa Snowden una conocida frase de Thomas Jefferson en 1798 y coloca a la encriptación en el mismo lugar en que el padre fundador había apelado a la Constitución: «Son palabras de la historia: no hablemos más de la fe en el hombre; atémoslo con las cadenas de la criptografía para que no haga travesuras» (Greenwald, 2014b, p. 38).

\section{DILEMAS PERIODÍSTICOS: MEDIADORES DE LA IN- FORMACIÓN EN LA ENCRUCIJADA DEL SECRETO Y LA TRANSPARENCIA}

\subsection{Periodismo profesional frente a transparencia informativa des-intermediada}

A diferencia de Wikileaks o Julian Assange, entre otros famosos filtradores anteriores, Snowden se plantea desde el comienzo contactar con profesionales escogidos de los medios de comunicación para ofrecerles los documentos reservados que testifican prácticas ilegales de vigilancia secreta masiva, llevadas a cabo por la NSA para el gobierno de los Estados Unidos de Norteamérica. Las revelaciones del ciudadano cosmopolita Snowden se producen deliberadamente a través de unos cuantos periodistas que él escoge por su perfil previo de compromiso con los derechos humanos: Laura Poitras (Citizen Four) y Glenn Greenwald (No Place to Hide). Frente a la práctica del desvelamiento indiscriminado de documentos secretos o confidenciales llevada a cabo por Wikileaks, ejemplo paradigmático de lo que se ha calificado como sueño de la «des-intermediación», ligado a la exigencia de transparencia (Innerarity, 2013, pp. 89-108), Snowden practica una filtración ajustada a una deliberada apuesta por el papel inter-mediador de los medios y de sus profesionales, entre los datos y un "público" o esfera pública, que se sobreentiende mundial, al que con frecuencia apela Snowden como destinatario de sus esfuerzos después de abandonar la NSA.

En su papel de redactores de las crónicas que dan forma y sentido al conglomerado de los documentos desvelados por los filtradores (Landert y Miscione, 2017), periodistas como Greenwald no actúan como simples altavoces ni delegados de Snowden. Al contrario, lo que este pretende es que sean justamente los profesionales de los medios -en el ejercicio de una responsabilidad con "el público" que él les atribuye principalmente a ellos- quienes decidan qué archivos son de interés general y cuál es el modo más apropiado de presentarlos. En el ejercicio responsable de este papel, se le plantean a la deontología periodística algunos dilemas éticos en su relación con esos "valientes filtradores" a los que Greenwald dedica su libro. Mucho se ha escrito sobre los cambiantes roles de los medios y del periodismo después de Wikileaks y de Snowden (Bell, Owen, Khorana y Hedrichsen, 2017; Landert y Miscione, 2017). Como señalan algunos trabajos recogidos en esta última publicación, en este mundo interconectado hay algunas habilidades nuevas que deberían adquirir los periodistas, que tienen inseparablemente una doble dimensión, instrumental y moral. Por ejemplo, estar entrenados en la encriptación de las comunicaciones para contribuir a la seguridad de sus informantes y mantener a salvo la confidencialidad de las fuentes. En estos tiempos en que, frente a la concentración de los medios, se invoca la necesidad de un "quinto poder" más directamente vinculado a la ciudadanía y a los nuevos medios digitales, el asunto Snowden revitaliza aquella imagen cuasi-mítica del intrépido reportero, de la prensa como "four state" (Greenwald, 2014a, pp. 210-247) y su coraje en el compromiso con el derecho a la información. "Fuentes valientes van a requerir de periodistas valientes y de publicaciones valientes" (Shirky, 2017, 7 febrero); si bien persiste la sombra de la crisis de los medios escritos en esta nueva era digital (Urich, 2015).

Así, pues, frente a la actual simpatía por la desintermediación, denunciada por Daniel Innerarity (2013), de las movilizaciones sociales de nuevo cuño, al considerar que la participación política, el acceso a la información o la reivindicación de "transparencia" solo se pueden llevar a cabo del modo más directo e in- 
mediato, esto es, lo menos mediatizado posible (por ejemplo, la calle frente a la política profesional, el periodismo ciudadano frente al de los grandes medios), Snowden cree en la mediación de los medios, en el paso por la mediaticidad y la intermediación mediática, de profesionales competentes, cívicamente valientes y responsables. Así se expresa tanto en la película de Laura Poitras como en la entrevista para La Sexta, en términos que suenan muy concordes con el análisis del fenómeno de Wikileaks que hace Innerarity (2013, pp. 95-97). Snowden representa una Ilamativa discrepancia con la tendencia emergente en las nuevas esferas públicas, configuradas por los medios digitales, que se caracterizan por la posibilidad de participación más plural y diversa de nuevos mediadores informativos frente a aquel clásico papel periodístico de gatekeepers de la noticia, que otorgaban o denegaban el acceso a la publicidad. Siendo hoy Edward Snowden un icono de la potencialidad política de los nuevos medios digitales, de las nuevas tecnologías en el activismo social y político, del hackactivismo o la ética hacker, de la firmeza en la exigencia de transparencia desde los nuevos medios, tiene mayor relevancia que precisamente él se apunte a una concepción de la esfera pública y de la esfera mediática, no tan des-mediatizada, ni tan des-inter-mediatizada.

\subsection{Transparencia informativa y protección del ano- nimato de las fuentes}

La transparencia podría ser cuestionada como una forma, quizá extremada, de reclamar el imperativo de publicidad como principio incondicional. En esto los medios de comunicación y el periodismo tienden a ser implacables. Es su obligación, en cierto modo, aunque ello a veces les ponga en situaciones difíciles. El propio Greenwald se enfrenta con este tipo de dilema en su primera conversación cara a cara con Snowden, que Laura Poitras registra audiovisualmente para lo que luego será CitizenFour: «Había una disonancia cognitiva entre, por un lado, reunirnos con una fuente que para el gobierno de Estados Unidos había cometido delitos graves y, por otro, filmarlo todo" (Greenwald, 2014b, p. 54). Por eso, contando con que Snowden podría ser detenido en cualquier momento, «mi máxima prioridad era saberlo todo acerca de Snowden: [...] En segundo lugar, estaba decidido a averiguar si era sincero y cooperativo o si estaba ocultando cosas importantes sobre quién era y lo que había hecho". Al interrogar entonces a Snowden, dice Greenwald haberse sentido, no tanto en su papel de informador cuanto más bien en su otra faceta de abogado, que quiere comprobar la sinceridad de los testigos. Dado que Snowden se presentaba como un filtrador altruista, el periodista también se mantiene «muy escéptico porque sabía que la credibilidad de todo lo que íbamos a hacer dependía de la fiabilidad de las afirmaciones de Snowden acerca de quién era» (Greenwald, 2014b, p. 56). El peligro de esta incondicional exigencia mediática de transparencia -dirigida ahora no al poder público, sino al denunciante de prácticas estatales fraudulentas- radicaba en que la atención se desviase desde el «asunto» mismo (documentos secretos, vigilancia amenaza la privacidad) hacia la persona o personalidad de Snowden, justo lo que él se temía (véase CitizenFour, minuto 23) y lo que sucedió, pese a todo, cuando se le motejó de narcisista en ciertos medios (CBS News, New York Times). Una total transparencia -cuando se le demanda a la persona particular- arriesga ser totalitaria, aunque se la exija en nombre del derecho y la libertad de información, ya la reclame el cuarto o el quinto poder. Aquí adquieren pleno sentido propuestas equilibradas, como la reciente y argumentada reivindicación de una matizada «transparencia sostenible» (Mingo Rodríguez, 2016).

\section{ALGUNAS LECCIONES DEL ASUNTO SNOWDEN PARA LA ÉTICA DE LA COMUNICACIÓN EN LA ERA DIGITAL}

En suma, ante las tensiones analizadas en el asunto Snowden entre transparencia y secreto, anonimato y visibilidad, se hace necesario recordar la normatividad de una delimitación entre lo público y lo privado en todos los regímenes políticos y la exigencia de un contrapeso en forma de libertades de información e investigación, frente a la tendencia de los Estados, incluso de los democráticos y de Derecho, a delimitar su terreno de juego con cierta política de arcano (Gerhardt, 2012, p. 138). Al mismo tiempo, este principio de publicidad, equilibrado con el respeto a la privacidad y el secreto personal, cuando se aplica al papel intermediador de los medios, se traduce, a partir de los dilemas encontrados en el tratamiento periodístico del asunto Snowden, en la necesidad de sopesar con cuidado los límites de la exigencia de transparencia.

En esto, el meticuloso proceder del propio Edward Snowden con los cerca de millón y medio de documentos altamente clasificados que robó a la NSA se constituye en ejemplo de esta ética para la ciudadanía y para los medios. Frente a la actitud, que cabe calificar cuanto menos de descuidada con la información personal o sensible para la seguridad por parte del hackactivismo y la transparencia a ultranza de Wiki- 
leaks, al poner directamente, sin mediación alguna, a la luz pública los datos que recibe, Snowden tomó sus precauciones para decidir qué documentos podía difundir, en principio unos doscientos mil, y al final parece que muchísimos menos (Holmes, 2018, pp. 157-158 y pp. 160-162). Y además lo hizo, evitando la tentación de la des-inter-mediación, en colaboración con periodistas de su confianza que participaron desde el principio en el proceso de seleccionar qué y cómo contar al público lo que realmente era de interés público, de todo lo que la información masivamente filtrada por Snowden podía revelar.

Una cierta ética del cuidado de la comunicación -en sus múltiples sentidos- se dibuja aquí como necesaria, empezando por la precaución de cada usuario de las redes sociales digitales, con los propios datos, la atención a lo que dice, cómo, a quién, así como la cautela y el trato esmerado a la información de y sobre las personas, por parte de quienes desde el principio, en este mundo de datos masivos, filtraciones, bulos, falsas noticias, tienen la misión de trabajar para el público, no para los poderes ni para los gobiernos: la profesión periodística (Tortosa, 2018). No es gratuito ni casual que la salvaguarda de la privacidad personal, frente a las maniobras secretas de determinadas corporaciones estatales o empresariales para saltársela, sea el pilar sobre el que se pueda construir como noticia de relevancia mundial el caso Snowden. Es, en efecto, una cuestión fundamental de democracia, derechos humanos, dignidad y libertad. Y ello dota con toda razón a la privacidad de un gran empuje movilizador de la opinión y del activismo social. En definitiva, se trata sobre todo, como nos interpela Snowden, de qué sociedad, de qué mundo queremos hacer entre todos. La privacidad que hemos de redescubrir después de su campaña de relevaciones no es un asunto meramente particularista, de intimidad o intereses individuales, sino un verdadero valor público, un auténtico bien común del que cuidar, atendiendo a la importancia de las relaciones, de la interdependencia, de la vulnerabilidad y la seguridad, no de los sistemas informáticos o de cualquier tipo, sino de los seres humanos vivientes de carne y hueso (Lyon, 2015, pp. 99-102, 128,136-137). En términos sociales, culturales, éticos y políticos, ni la privacidad, ni la publicidad están solas, como bienes a defender, a proteger y a cultivar. Las tensiones cruzadas entre principios y valores (privacidad, publicidad, secreto, transparencia, anonimato, visibilidad, confidencialidad, derecho a la información) que el análisis de los dilemas de la esfera pública, del activismo y de los medios esbozado en este trabajo pone de manifiesto apuntan a que esta ética del cuidado de y con la comunicación ha de ser también una ética de la responsabilidad, centrada en el respeto a las personas.

\section{AGRADECIMIENTOS}

Este trabajo recoge resultados de estancias de investigación en la Universidad Humboldt de Berlín financiadas por una beca José Castillejo (CAS14/00398) del Ministerio de Educación, Cultura y Deporte, y por el Plan Propio de la Universidad de Almería. Ha contado, además, con soporte financiero del Proyecto Sostenibilidad de la atención a las personas en situación de dependencia: experiencias y dilemas en el diseño de tecnocuidados (P18-TP-2625). Agradezco sus valiosas sugerencias a uno de los revisores anónimos del manuscrito y a mis colegas de la Universidad de Almería, Juan Sebastián Fernández Prados y María Teresa Martín Palomo.

\section{BIBLIOGRAFÍA}

Arendt, H. (1958/1998). The Human Condition. Chicago / London: University of Chicago Press.

Arquembourg, J. (1998). L'incendie du tunnel sous la Manche. Les deux faces d'une médiation journalistique. Réseaux, 16 (91). pp. 135-149. https://doi. org/10.3406/reso.1998.3211

Arquembourg, J. (2009). Who did the tsunami happen to? An analysis of the media account of events as part of a process in collective understanding. Global Media and Communication, 5 (3), pp. 389-404. https://doi. org/10.1177/1742766509356487
Ballenthien, J., Hensel, A., Hoeft, C., Ulbrich, M., Rohde, M., Rohwerder, J. y Urich, K. (2015). Zwischen Sichtbarkeit und Anonymität. Protest, Bewegung und digitale Kultur. Forschungsjournal Soziale Bewegungen, 28 (3), pp. 3-7. https:// doi.org/10.1515/fjsb-2015-0302

Bauman, Z. y Lyon, D. (2013). Vigilancia líquida. Buenos Aires: Paidós.

Bell, E., Owen, T., Khorana, S. y Henrichsen, J. R. (eds.). (2017). Journalism after Snowden: the future of the free press in the surveillance state. New York: Columbia University Press. https://doi. org/10.7312/bell17612
Benhabib, S. (1996). The Reluctant Modernism of Hannah Arendt. London / New Delhi / Thousand Oaks: SAGE.

Borradori, G. (2016). Between transparency and surveillance: Politics of the secret. Philosophy and Social Criticism. 42 (4-5), pp. 456-464. https://doi. org/10.1177/0191453715623321

Callejo, J. (2003). El silencio: núcleo ético de la comunicación. Comunicar. Revista Científica de Comunicación y Educación, 20, pp. 173-177. https://doi. org/10.3916/C20-2003-25

Freudenschuß, M. (2015). Paradoxe Dynamik. Aktivismus zwischen Anonymität 
und Sichtbarkeit. Forschungsjournal Soziale Bewegungen, 28 (3), pp. 55-61. https://doi.org/10.1515/fjsb-2015-0308

Ganz, K. (2015). Zehn Jahre Netzbewegung. Konflikte um Privatheit im digitalen Bürgerrechtsaktivismus vor und nach Snowden. Forschungsjournal Soziale Bewegungen, 28 (3), pp. 35-45. https:// doi.org/10.1515/fjsb-2015-0306

Gerhardt, V. (2012). Öffentlichkeit. Die politische Form des Bewusstseins. München: Verlag C. H. Beck. https://doi. org/10.17104/9783406633041

Greenwald, G. (2014a). No Place to Hide: Edward Snowden, the NSA, and the US Surveillance State. New York: Metropolitan Books.

Greenwald, G. (2014b). Sin un lugar donde esconderse. Barcelona: Ediciones B.

Han, B. (2014). En el enjambre. Barcelona: Herder. https://doi.org/10.2307/j.ctvt9k4gh

Holmes, D. E. (2018). Big Data. Una breve introducción. Barcelona: Antoni Bosch editor.

Hölscher, L. (1979). Öffentlichkeit und Geheimnis. Eine begriffsgeschichtliche Untersuchung zur Entstehung der Öffentlichkeit in der frühen Neuzeit. Stuttgart: Klett-Cotta.

Innerarity, D. (2013). Un mundo de todos y de nadie. Piratas, riesgos y redes en el nuevo desorden global. Barcelona: Paidós.
Koselleck, R. (1959). Kritik und Krise. Eine Studie zur Pathogenese der bürgerlichen Welt. Freiburg im Breisgau / München: Karl Alber Verlag.

Landert, D. y Miscione, G. (2017). Narrating the stories of leaked data: The changing role of journalists after Wikileaks and Snowden. Discourse Context \& Media, 19, pp. 13-21. DOI: https://doi. org/10.1016/j.dcm.2017.02.002

Lyon, D. (2015). Surveillance after Snowden. Cambridge: Polity Press.

Mingo-Rodríguez, A. de (2016). Vivir a la vista de todos. Ensayos sobre humanismo y transparencia sostenible. Sevilla: Fénix Editora.

Ochs, C. (2015). "Selbstdatenschutz», oder: Kollektive Privatheitspraktiken als politisches Handeln in digitalen Öffentlichkeiten. Forschungsjournal Soziale Bewegungen, 28 (3), pp. 45-54. https://doi.org/10.1515/fjsb-2015-0307

Shirky, C. (2017, 7 febrero). Political Journalism in a Networked Aged. [Reseña de Journalism After Snowden: the future of the free press in the surveillance state de $\mathrm{E}$. Bell, T. Owen, S. Khorana y J. R. Henrichsen (eds.).] Nieman Reports. [En línea]. Disponible en: http://niemanreports.org/ tag/journalism-after-snowden/

Simmel, G. (1993). Gesamtausgabe (vol. 8. Aufsätze und Abhandlungen 19011908). Frankfurt am Main: Suhrkamp.
Simmel, G. (1986). Sociología 1. Estudios sobre las formas de socialización. Madrid: Alianza.

Tortosa, J. (2018). Periodistas. El arte de molestar al poder. Barcelona: Roca Editorial.

Urich, K. (2015). Der Fall Snowden im Spiegel der Literatur. Forschungsjournal Soziale Bewegungen, 28 (3), pp. 115-117.

Whitaker, R. (1999). The End of Privacy. How Total Surveillance is Becoming a Reality. New York: The New Press.

Wegener, B. W. (2006). Der geheime Staat. Arkantradition und Informationsfreiheit. Göttingen: Morango.

\section{Otros recursos}

La Sexta (2016, 13 de marzo). Ana Pastor entrevista en exclusiva a Edward Snowden en El Objetivo. Entrevista completa a Edward Snowden en El Objetivo (versión extendida en V.O.). [En línea]. Disponible en: https://www.lasexta. com/programas/el-objetivo/noticias/ entrevista-completa-edward-snowdenobjetivo-version-extendida_201603135 72398a04beb28d446ffed10.htm

Wilutzky, D. (productor) y Poitras, L. (directora) (2014). CitizenFour. [Cinta cinematográfica]. EE.UU., Alemania, Reino Unido: Praxis Films. 